\title{
Anticipated synchronization in neuronal motifs
}

\author{
Fernanda S Matias ${ }^{1,2^{*}}$, Leonardo L Gollo ${ }^{2,3}$, Pedro V Carelli ${ }^{1}$, Mauro Copelli ${ }^{1}$, Claudio R Mirasso ${ }^{2}$ \\ From Twenty Second Annual Computational Neuroscience Meeting: CNS*2013 \\ Paris, France. 13-18 July 2013
}

Anticipated synchronization (AS) is a counterintuitive stable dynamical regime discovered by Voss in the last decade [1]. It consists in the stable synchronized regime between two identical autonomous dynamical systems coupled (unidirectionally) in a master-slave (MS) configuration, if the slave also receives a negative delayed selffeedback. In such regime the slave predicts the master. Although AS has shown to be stable in a variety of theoretical studies [1-3] and in experimental observations [4] with semiconductor lasers and electronic circuits, studies in biological and neuronal systems are still lacking.

The first verification of AS in neuronal models was done between two FitzHugh-Nagumo neurons coupled in a MS configuration with negative delayed self-feedback and driven by white noise [2]. In neuronal networks, AS means that the slave (post-synaptic) neuron can fire spikes right before the master (pre-synaptic) neuron. However the slave delayed self-feedback that induces the anticipated synchronization as suggested by Voss is unrealistic in neuronal circuitry.

Recently, it has been shown that in a biologically plausible neuronal model, the self-feedback can be replaced by an inhibitory loop mediated by an interneuron and chemical synapses [5]. This master-slave-interneuron motif exhibits the usual delay synchronized (DS) regime for small values of the inhibitory synaptic conductance $(g)$ as well as the AS regime for larger $g$. Moreover, the time delay between consecutive spikes of the master and the slave neurons is a function of the synaptic conductances. Both regimes were shown to be stable for a large set of parameters, different external currents, structural motif and neuron models [6]. Here we investigate the robustness of AS for others motifs, larger populations, neuronal heterogeneity and noise. In all the situations the transition from DS to AS is continuous and smooth.

\section{Acknowledgements}

We acknowledge financial support from CNPq, CAPES, FACEPE, PRONEX, PRONEM, INCeMaq, proyecto FISICOS (MICCIN-FEDER) and Govern de les Illes Balears.

\section{Author details}

${ }^{1}$ Departmento de Física, Universidade Federal de Pernambuco, Recife, Pernambuco 50670-901, Brazil. ${ }^{2}$ Instituto de Física Interdisciplinar y Sistemas Complejos, CSIC-UIB, Campus Univesitat de les Illes Balears E-07122 Palma de Mallorca, Spain. ${ }^{3}$ Systems Neuroscience Group, Queensland Institute of Medical Research, Brisbane, QLD 4006, Australia.

Published: 8 July 2013

\section{References}

1. Voss HU: Anticipating chaotic synchronization. Phys Rev E 2000, 61:5115-5119.

2. Ciszak M, Calvo O, Masoller C, Mirasso CR, Toral R: Anticipating the response of excitable systems driven by random frocing. Phys Rev Lett 2003, 90:204102-204104.

3. Masoller C, Zanette DH: Anticipated synchronization in coupled chaotic maps with delays. Physica A 2001, 300:359-366.

4. Sivaprakasam S, Shahverdiev EM, Spencer PS, Shore KA: Experimental demonstration of anticipating synchronization in chaotic semiconductor lasers with optical feedback. Prys Rev Lett 2001, 87:154101.

5. Matias FS, Carelli PV, Mirasso CR, Copelli M: Anticipated synchronization in a biologically plausible model of neuronal motif. Phys Rev E 2011, 84:021922-021929.

6. Matias FS, Gollo LL, Carelli PV, Copelli M, Mirasso CR: Anticipated synchronization in neuronal network motifs. AIP Conference Proceedings 2013, 1510:210-212.

doi:10.1186/1471-2202-14-S1-P275

Cite this article as: Matias et al:: Anticipated synchronization in neuronal motifs. BMC Neuroscience 2013 14(Suppl 1):P275.

\footnotetext{
* Correspondence: fernandasm@df.ufpe.br

'Departmento de Física, Universidade Federal de Pernambuco, Recife,

Pernambuco 50670-901, Brazil

Full list of author information is available at the end of the article
}

(c) 2013 Matias et al; licensee BioMed Central Ltd. This is an Open Access article distributed under the terms of the Creative Commons 